\title{
Reliability theory application for building structures reliability determination
}

\author{
Nadezhda Chiganova ${ }^{1, *}$ \\ ${ }^{1}$ National research Moscow state university of civil engineering, Yaroslavskoye sh. 26, 129337 \\ Moscow, Russia
}

\begin{abstract}
Nowadays the determination of building structures and their components reliability in the variable mode is an important task. These tests are as follows: the batch of products intended for test are first tested in the first mode, and then in the second mode. A certain moment of time for mode switching is selected randomly by a specified law. The product is tested in the variable two-stage mode. During testing, moments of time of product operation in the first and second modes are registered. Two experimentation programs that differ in the way of mode switching are proposed: program tests and dynamic tests. Examples of the methodology application to the determination of the product failure rate were discussed. Besides, the approximate calculation method of the reliability characteristics in the second mode was discussed.
\end{abstract}

\section{Introduction}

The solution to many of the reliability theory problems [8] requires building structures and their components (hereinafter - "products") testing in the variable mode. Such problems include, for example, stress test problems, reliability calculation in the variable mode [1-4], life utilization principles validation of the products [5], replacement of the operation modes with laboratory ones [6].

Tests in variable regime are as follows. The batch of products intended for test are first tested in the mode $\varepsilon_{1}$, and then in the mode $\varepsilon_{2}$. Moment $\tau$ of time for mode switching is selected randomly by a specified law $\mathrm{H}(\mathrm{t})=\mathrm{P}(\mathrm{t}<\tau)$. The product is tested in the variable twostage mode:

$$
\varepsilon=\left\{\begin{array}{l}
\varepsilon_{1}, 0 \leq \mathrm{t}<\tau \\
\varepsilon_{2}, \tau \leq \mathrm{t}<\infty
\end{array}, \text { where } \tau \text {-random variable distributed according to the law } \mathrm{H}(\mathrm{t}) .\right.
$$

The article studies the problem of determining the building structures and their components reliability performance in mode $\varepsilon_{1}$ and $\varepsilon_{2}$ based on the results of the tests described. Let us set $\xi_{\mathrm{i}}$ and $\tilde{\xi}$ product failure time in the modes $\varepsilon_{i}$ and $\tilde{\varepsilon}$, respectively and through $F_{i}$ and $\tilde{F}$ their distribution function, i.e. $F_{i}(t)=P\left(\xi_{i}<t\right), \mathrm{i}=1.2, \tilde{F}(t)=P(\tilde{\xi}<$ t).

\footnotetext{
* Corresponding author: chiganovanm.mgsu@,gmail.com
} 


\section{Determination of the building structures and their components reliability performance}

Note that there is the following relationship between random variables $\theta_{1}, \theta_{2}$ and $\xi_{\mathrm{i}}, \tilde{\xi}$ : $\theta_{1}=\min \left\{\xi_{i}, \tau\right\}, \theta_{2}=\max \{0, \tilde{\xi}-\tau\}$.

As the characteristics $\theta_{1}, \theta_{2}$ and $\tilde{\xi}$ are observed during the test, after the experimental test distribution functions $\tilde{F}(t), \theta_{i}(t)=P\left(\theta_{i}<t\right), i=1.2$ and $S(t)=P\left(\theta_{2}>t / \xi_{i}>\tau\right)$ can be restored. $\mathrm{S}(\mathrm{t})$ function is the conditional probability that the product not failed in the first mode during its testing on the program $\tilde{\varepsilon}$, will operate in the second mode for no longer than t. Subsequently we distinguish between two experimentation programs that differ in the way of mode switching.

A) Program tests. They consist in the fact that before the experiments start they are specified by the distribution function $H(t)$. Further, $n$ random values $\mu_{1}, \mu_{2}, \ldots, \mu_{n}$, evenly distributed by the law are determined by the random number tables corresponding to the number $\mathrm{n}$ of test products. Values $\tau_{i}=H^{-1}\left(\mu_{i}\right), i=1,2, \ldots, n$ are calculated, where $\mathrm{H}^{-1}-$ inverse $\mathrm{H}$ function. Product number $\mathrm{i}$ is tested first in the mode $\varepsilon_{1}$ for a time $\tau$, then, if it does not fail - in the mode $\varepsilon_{2}$. Any function $H$ that has the probability density $h(t)>0$ at all $\mathrm{t}>0$ can be chosen.

B) Dynamic tests. In these tests, the mode switching is carried out only in the product failure times. Briefly the test sequence will be as follows. At first, all products are tested in the mode $\varepsilon_{1}$. Let's assume that the first failure occurred in the time $t$. Then randomly selected not failed product is switched to the second mode, and the remaining n-2 continue to be tested in the mode $\varepsilon_{1}$ as long as one of them fails. Assume that it happens in the moment of time $\tau_{2}$. Again, randomly selected not failed product is switched to the second mode, and the remaining n-4 continue to be tested in the first mode, etc. It is evident that in this way each product will be tested in the variable mode of (I) type.

So, we will proceed from the fact that similar tests were carried out and, based on their results, distributions $\mathrm{Q}_{i}, \mathrm{~S}$ were obtained. First let us set a goal to find the distribution of products failure-free operation in the first mode $\bar{F}_{1}(t)=1-F_{1}(t)$ by received $\mathrm{Q}_{\mathrm{i}}, \mathrm{S}$.

1. Let the experiment be carried out under program A) and based on its results function $H, Q_{i}, S$ are obtained. It follows from the definition of random variable $\theta_{1}$ that

$$
\begin{aligned}
\theta_{1}(\mathrm{e})= & P\left(\theta_{1}<t\right)=P\left(\min \left\{\xi_{1}, \tau\right\}<t\right)=1-P\left(\xi_{1}>t, \tau<t\right) \\
& =1-\left[1-F_{1}(t)\right][1-H(t)]=1-\bar{F}_{1}(t) \bar{H}(t), \bar{Q}_{1}(t) \\
& =\bar{F}_{1}(t) \bar{H}(t)
\end{aligned}
$$

From formula (2) we obtain the reliability function in the first mode

$$
\bar{F}_{1}(t)=\frac{\bar{Q}_{1}(t)}{\bar{H}(t)}, \bar{F}_{1}(t)=1-F_{1}(t)=P\left(\xi_{1}>t\right)
$$

We shall write the numerical characteristics of this distribution: mathematical expectation and variance of random variable:

$$
M(\xi)=\int_{0}^{\infty} \bar{F}_{1}(t) d t=\int_{0}^{\infty} \frac{\bar{Q}_{1}(t)}{\bar{H}(t)} d t, D\left(\xi_{1}\right)=M\left(\xi_{1}^{2}\right)-M^{2}(\xi)
$$

It should be noted that

$$
M\left(\xi_{1}^{2}\right)=2 \int_{0}^{\infty} t \bar{F}_{1}(t) d t=2 \int_{0}^{\infty} \frac{\bar{Q}_{1}(t)}{\bar{H}(t)} d t
$$


2. Let the tests be carried out under the programs B). In this case the switching distribution $\mathrm{H}(\mathrm{t})$ will not be known because it coincides with $F_{1}(t)$. Applying $H=F_{1}$ in $(2)$, we obtain

$$
F_{1}(t)=\sqrt{\bar{Q}(t)}
$$

Formulas (2) and (3) show that in order to determine the product reliability characteristics in the first mode it is sufficient to find the lifetime distribution $Q_{l}$ based on the test conducted results $\theta_{1}$.

Although formulas (2) and (3) are simple, their use in practice may give rise to some difficulties. In fact, after the experimental study failure time will be known, and it is difficult to determine the distribution type by this data.

Therefore, $Q_{1}$ functions for a given failures distribution of $F_{1}$ and $H$ are a matter of interest.

\section{Examples}

1. Let the products fail in the first mode exponentially $F_{1}(t)=1-e^{-\lambda_{1} t}$ and switching points are also set exponentially $H(t)=1-\exp \left(-\lambda_{2} t\right)$. Then $\bar{Q}_{1}(t)=\bar{F}_{1}(t) H(t)=$ $\exp \left[-\left(\lambda_{1}+\lambda_{2}\right) t\right]$.

$$
M\left(\theta_{1}\right)=\int_{0}^{\infty} \bar{Q}_{1}(t) d t=\int_{0}^{\infty} \exp \left[-\left(\lambda_{1}+\lambda_{2}\right) t\right] d t=\frac{1}{\lambda_{1}+\lambda_{2}}
$$

We deduce from the last equation the failure rate in the first mode:

$$
\lambda_{1}=\frac{1-\lambda_{2} M\left(\theta_{1}\right)}{M\left(\theta_{1}\right)}
$$

2. Failures time is distributed by Poisson law: $F_{1}(t)=t^{n} e^{-t} / n !$ and switching times are chosen exponentially: $H(t)=1-\exp (-\lambda t)$. In this case:

$$
\begin{gathered}
\bar{Q}_{1}(t)=e^{-\lambda t}\left(1-\frac{t^{n} e^{-t}}{n !}\right), M\left(\theta_{1}\right)=\int_{0}^{\infty}\left[e^{-\lambda t}-\frac{t^{n} e^{-t(1+\lambda)}}{n !}\right] d t=\frac{1}{\lambda}-\frac{1}{(1+\lambda)^{n+1}}, \text { where } \\
(1+\lambda)^{n+1}=\frac{\lambda}{1-\lambda M\left(\theta_{1}\right)}, n=\log _{(\lambda+1)}\left[\frac{\lambda}{1-\lambda M\left(\theta_{1}\right)}\right]-1 .
\end{gathered}
$$

\section{Determination of the failures distribution in dynamic regime}

Let us now determine the failures distribution $F_{2}$ in the second mode. Note that the $F_{2}$ function can not be clearly determined according to $Q_{i}$ and $H$ data obtained after the tests. It is necessary to have additional information on the products life utilization in the variable mode.

Currently, many product functional loss models were proposed [1-5]. We select the most widespread model proposed by N.M. Sedyakin [6]. According to this model, the products reliability depends on its life span value in the past and does not depend on the manner (mode, time) of the life span end. N.M. Sedyakin proposes to select the cumulative failure rate as a life span measure. Sedyakin principle mathematically may be written as follows.

If $P\left(t_{1}, \varepsilon_{1}\right)=P\left(t_{2}, \varepsilon_{2}\right)$, then the equality $P\left(t, \varepsilon_{2} / t_{1}, \varepsilon_{1}\right)=P\left(t, \varepsilon_{2} / t_{2}, \varepsilon_{2}\right)$ is valid. Here $P\left(t, \varepsilon_{2} / t_{i}, \varepsilon_{i}\right), i=1,2$, it means the probability of failure-free operation for a time $t$ in the 
mode $\varepsilon_{2}$ for such products which have been already operated in the mode $\varepsilon_{i}$ without a failure for a time $t_{i}$.

Based on Sedyakin principle it is easy to solve the inverse problem of determining distribution functions $F_{1}, F_{2}$ and $H S(t)=P\left(\theta_{2}>t / \xi_{1}>\tau\right)$. In fact, $S(t / x)=P\left(\theta_{2}>\right.$ $\left.t / \xi_{1}>\tau, \tau=x\right)$.

By the total probability formula

$$
S(t)=P\left(\theta_{2}>t / \xi_{1}>\tau\right)=\int_{0}^{\infty} P\left(\theta_{2}>t / \xi_{1}>\tau, \tau=x\right) d H(x)=\int_{0}^{\infty} S(t / x) d H(x)
$$

The conditional distribution $S(t / x)$ is uniquely adjusted by $F_{1}$ and $F_{2}$ functions based on Sedyakin principle. Let us denote $\hat{x}$ as the solution of equation

$$
F_{1}(x)=F_{2}(\hat{x})
$$

According to Sedyakin principle, product testing in the mode $\varepsilon_{1}$ for a time x equivalent to test in the mode $\varepsilon_{2}$ and duration $\hat{x}$. Therefore,

$$
S(t / x)=P\left(\xi(\widetilde{\varepsilon})>t+x / \xi_{1}>x\right)=P\left(\xi_{2}>t+\hat{x} / \xi_{2}>x\right)=\frac{\bar{F}_{2}(t+\hat{x})}{\bar{F}_{2}(\hat{x})}
$$

After substituting (5) and (6) in (4) we obtain

$$
S(t)=\int_{0}^{\infty} \frac{\bar{F}_{2}(t+\hat{x})}{\bar{F}_{2}(\hat{x})} d H(x)
$$

Or by replacing $\hat{x}=\bar{F}_{2}^{-1}\left(\bar{F}_{1}(x)\right)$ in accordance with (6)

$$
S(t)=\int_{0}^{\infty}\left\{\bar{F}_{2}\left[t+\bar{F}_{2}^{-1}\left(\bar{F}_{1}(x)\right)\right] / \bar{F}_{1}(x)\right\} d H(x)
$$

Thus, the distribution $\bar{F}_{2}$ is defined as the solution of the integral equation (7).

In case of dynamic tests $H=F_{1}$ formula (7) can be simplified by introducing a new variable of integration $\xi=\bar{F}_{1}(x)$, that enables to reduce (7) to the form

$$
S(t)=\int_{0}^{1}\left\{\bar{F}_{2}\left(t+\bar{F}_{2}^{-1}(\xi) / \xi\right\} d \xi\right.
$$

The solution of this integral equation poses a challenge, because the element of integration includes the inverse function $\bar{F}_{2}^{-1}$. This disadvantage can be eliminated if instead of distribution $F_{2}$ the failure rate $\lambda_{2}(x)=-\frac{d \ln F_{2}}{d x}$ will be found. It is easy to verify, if we pass to a new variable of integration $\eta=\bar{F}_{2}^{-1}(\xi)$.

Then

$$
\begin{aligned}
\xi=\bar{F}_{2}(\eta), & d \xi=-\lambda_{2}(\eta) \bar{F}_{2}(\eta) d \eta \\
S(t) & =\int_{0}^{\infty} \exp \left[-\int_{0}^{t+\eta} \lambda_{2}(x) d x\right] \lambda_{2}(\eta) d \eta
\end{aligned}
$$

and,

hence,

In general, the solution of equation (7) may be carried out by a computer.

Let us consider the approximate calculation method of the reliability characteristics in the second mode by the known functions $S, \mathrm{Q}_{i}$ and $H$. Let $Q(t / x)=P\left(\xi_{2}<t / \xi_{1}=x\right)-$ conditional distribution of failures in the mode $\varepsilon_{2}$ of product, which in the first mode would have operated exactly the time $\mathrm{x}$. By setting the function $\mathrm{Q}$ distributions of $\mathrm{F}_{2}$ and $\mathrm{S}$ are clearly determined. Indeed, 


$$
F_{2}(t)=\int_{-\infty}^{\infty} Q(t / x) d F_{1}(x)
$$

Assuming $\mathrm{Q}$ as a constant during the products tests in the mode $\varepsilon_{1}$, we can write

$$
\begin{gathered}
S(t / x)=P\left(\xi_{2}\right. \\
\left.>t / \xi_{1}=x\right)=\int_{0}^{\infty}[1-Q(t / y)] \frac{d F_{1}(y)}{F_{1}(x)}=\frac{1}{F_{1}(x)} \int_{0}^{\infty} d F_{1}(y) \int_{0}^{\infty} \psi_{t}(z) d Q(z / x) \\
\text { where } \psi_{t}(z)=\left\{\begin{array}{l}
0, \mathrm{z}<t, \\
1, \mathrm{z} \geq \mathrm{t} .
\end{array}\right.
\end{gathered}
$$

By inserting $S(t / x)$ into (4) we obtain

$$
S(t / x)=\int_{0}^{\infty} \frac{d H(x)}{F_{1}(x)} \int_{x}^{\infty} d F_{1}(y) \int_{0}^{\infty} \psi_{t}(z) d Q(z / x)
$$

Or, changing the order of integration

$$
S(t / x)=\int_{0}^{\infty} d F_{1}(y) \int_{0}^{y} \frac{d H(x)}{F_{1}(x)} \int_{0}^{\infty} \psi_{t}(z) d Q(z / x)
$$

Let us set $\Pi(x)=\int_{0}^{\infty} d F_{1}(y) \int_{0}^{y} \frac{d H(\xi)}{F_{1}(\xi)}, I_{Q}^{t}(x)=\int_{0}^{\infty} \psi_{t}(z) d Q(z / x)$.

Then (9) can be presented as

$$
S(t)=\int_{0}^{\infty} I_{Q}^{t}(x) d \Pi(x)
$$

Since the bivariate function $\mathrm{Q}$ is not known and can not be determined in principle based on the conducted tests results (because the simultaneous failure time measuring of the same product in different modes is not possible), then $F_{2}$ distribution is ambiguously determined by setting the function $\mathrm{S}$. In this regard, let us find the lower $F_{2 *}$ and upper $\mathrm{F}_{2}{ }^{*}$ boundaries for $\mathrm{F}_{2}$ under condition (10), considering $F_{2}$ as functional of $\mathrm{Q}$. Let $M\left(F_{1}, F_{2}\right)$ and $R(S, \Pi)$ are set of all distributions $\mathrm{Q}$ that satisfy equations (8) and (10), respectively. In addition, let us introduce the set $L_{t}$ of all conditional distributions for which $I_{Q}^{t}(x)$ is a nondecreasing function $\mathrm{x}$ at any fixed $\mathrm{t}$.

Let's assume that $Q \cap_{t \in x} L_{t} \equiv L$. Physically, it means that the longer the product operates in the first mode, the more reliable in the mode $\varepsilon_{2}$ it is.

From the mathematical point of view, the evaluation of $F_{2}$ distribution is reduced to the variational problem associated with the determination of absolute minimum $F_{2 *}(t)=$ $\inf _{Q \in x} F_{2}(t)$ and absolute maximum $F_{2}^{*}(t)=\sup _{Q \in x} F_{2}(t)$ of $F_{2}$ functional on the set $\mathrm{X}=L \cap M$. Because functionals $F_{2}, I_{Q}^{t}, S(t)$ are linear in $\mathrm{Q}$, the duality principle is valid. According to this principle

$$
\begin{gathered}
{\left[\int Q_{*} d F_{1}=\inf _{Q \in x} \int Q d F_{1}\right]<=>\left[\int I_{Q} d \Pi=\inf _{Q \in x} \int I_{Q} d \Pi\right],} \\
{\left[\int Q^{*} d F_{1}=\sup _{Q \in x} \int Q d F_{1}\right]<=>\left[\int I_{Q} d \Pi=\sup _{Q \in x} \int I_{Q} d \Pi\right],} \\
Q_{*}, Q^{*} \in \mathrm{X} \cap S .
\end{gathered}
$$

This principle allows the functional extremums $S(t)$ to be found instead of the variational problem on the set $M \cap L$. Therefore, let us find $S_{*}(t)$ :

$$
S_{*}(t)=\inf _{Q \in x} \int_{0}^{\infty} I_{Q}^{t}(x) d \Pi(x), S^{*}(t)=\sup _{Q \in x} \int_{0}^{\infty} I_{Q}^{t}(x) d \Pi(x)
$$

Decision algorithm for this variational problem is given in [9]. According to this algorithm, let us form the probabilities ratio 


$$
\gamma(x)=d \Pi(x) / d F_{1}(x)=\int_{0}^{x}\left(1 / \bar{F}_{1}(\xi)\right) d H(\xi)
$$

Because $\gamma(x)$ is a non-decreasing function, then in virtue of Theorem (3) [9]

$$
Q_{*}(t / x)\left\{\begin{array}{l}
0, t<t_{*}(x) \\
1, t \geq t_{*}(x)
\end{array}, Q^{*}(t / x)=F_{2}(t)\right.
$$

Where $t_{*}(x)$ - solution of equation $F_{2}\left(t_{*}\right)=F_{1}(t)$.

By inserting (11) into (10) we obtain:

$$
\begin{gathered}
S(t)=\int_{0}^{\infty} d F_{1}(y) \int_{0}^{y} \frac{d H(x)}{\bar{F}_{1}(x)} x\left[t_{*}(x)-t\right], x(t)=\left\{\begin{array}{l}
0, t \leq 0 \\
1, t>0
\end{array}\right. \\
S(t)=\int_{0}^{\infty} d F_{1}(y) \int_{0}^{y} \frac{d H(x)}{\bar{F}_{1}(x)} \bar{F}_{2}(t)
\end{gathered}
$$

Because $\int_{0}^{\infty} d F_{1}(y) \int_{0}^{y} \frac{d H(x)}{\bar{F}_{1}(x)}=1$, from (13) it follows that $\bar{F}_{2}^{*}(t)=S(t)$.

By inserting $t_{*}(x)=F_{2}^{-1}\left[F_{1}(x)\right]$ into (12) we obtain

It is evident that $F_{2}^{-1}\left[F_{1}(x)\right] \geq t$.

$$
S(t)=\int_{0}^{\infty} d F_{1}(y) \int_{0}^{y} \frac{d H(x)}{\bar{F}_{1}(x)} x F_{2}^{-1}\left[F_{1}(x)-t\right]
$$

Then $x \geq F_{2}^{-1}\left[F_{1}(x)\right], S(t)=\int_{F_{1}^{-1}\left[F_{2}(t)\right]}^{\infty} d F_{1}(y) \int_{F_{1}^{-1}\left[F_{2}(t)\right]}^{\infty}\left(1 / F_{1}(x)\right) d H(x)$.

Let us change the order of integration.

$$
S(t)=\int_{F_{1}^{-1}\left[F_{2}(t)\right]}^{\infty}\left(1 / F_{1}(x)\right) d H(x) \int_{x}^{\infty} d F_{1}(y)=\int_{F_{1}^{-1}\left[F_{2}(t)\right]}^{\infty} d H(x)
$$

It follows that $S(t)=\bar{H}\left[F_{1}^{-1}\left(F_{2}(t)\right)\right]$ and $F_{2 *}(t)=F_{1}\left[H^{-1}(S(t))\right]$. In case of dynamic tests $H=F_{1}$ the formula will be $F_{2 *}(t)=S(t)$.

Thus, the distribution of failure-free products operation in the second mode is within the boundaries

$$
\bar{F}_{1}\left[H^{-1}(\bar{S}(t))\right] \leq \bar{F}_{2}(t) \leq S(t)
$$

Function $\bar{F}_{1}$ included in (14) is determined by formulas (2) and (3).

\section{Conclusions}

In the article of I.A. Ushakov "Is reliability theory still alive?" [8] the definite answer is given - it is alive. Today the reliability theory becomes increasingly important because the quality and reliability determination in the variable mode for building structures and their components is frequently required.

The results obtained in this article can simplify the determination of the quality and reliability of building structures and their components during testing modes switching.

\section{References}

1. B.V. Gnedenko, J.K. Beljaev, A.D. Solov'ev, Mathematical methods in reliability theory (Nauka, 2010) 
2. N.M. Chiganova, Natural and Technical Sciences 6, 14-17 (2015)

3. V. Medvedev, A. Pustovgar, Applied Mechanics and Materials 725-726, 337-382 (2015)

4. G.D. Kartashov, N.M. Chiganova, Journal of Mathematical Sciences 39, 2578-2588 (1987)

5. G.D. Kartashov, Elements and schemes accelerated testing (monograph) (Knowledge, 1990)

6. N.M. Sedjakin, Technical Cybernetics - USSR Academy of sciences proceedings 3, 80-87 (1968)

7. A.I. Kubarev, I.Z. Aronov, O.I. Teskin et al., Methodical guidelines. The reliability of the technique. Methods for assessing the reliability of indicators based on experimental data (Standards Publishing House - USSR Gosstandart, 1991)

8. I.A. Ushakov, Reliability: Theory \& Applications 2, 6-19 (2007)

9. G.D. Kartashov, Theory of Probability and its Applications 14(4), 595-611 (1969) 